\title{
Introduction to Exercise is Medicine Global Health Initiative
}

\author{
Mark Stoutenberg \\ (マイアミ大学医学部(University of Miami Miller School of Medicine))
}

The EIM initiative was first introduced in 2007 to advance the implementation of evidence-based strategies to elevate the status of physical activity (PA) in healthcare. To date, EIM has focused on building an infrastructure and raising awareness of the multiple health benefits of regular PA. EIM now has a presence in more than 40 countries globally, with Regional Centers established in North America, Latin America, Europe, Southeast Asia, China, Africa and Australasia. Recently, EIM has begun transitioning from its initial phase of infrastructure and awareness building to a phase of integration and program implementation into healthcare settings and surrounding communities. Through this presentation, we seek to increase the understanding about EIM and its implementation to better engage the vast array of professionals and resources - from practitioners to research scientists to fitness professionals - in the future development, expansion, and evaluation of EIM in the Japan. We will describe the specific tools that have been developed for the implementation of EIM into both clinical and community settings and the steps that can be taken to enhance the linkages between these sectors. Broad implementation of PA assessment, as clinical practice standard of care, and referral systems, leveraging community resources and professional networks, has the potential to improve PA at the population level and to be an important part of the solution for achieving national targets for the reduction of physical inactivity and related morbidity and mortality. 